\title{
Dopaminergic Modulation of Cortical Plasticity in Alzheimer's Disease Patients
}

\author{
Giacomo Koch*,',2, Francesco Di Lorenzo',2, Sonia Bonni', Viola Giacobbe', Marco Bozzali ${ }^{3}$, \\ Carlo Caltagirone ${ }^{1,2}$ and Alessandro Martorana ${ }^{2}$ \\ 'Non Invasive Brain Stimulation Unit, Department of Behavioural and Clinical Neurology, Santa Lucia Foundation IRCCS, Rome, Italy; \\ ${ }^{2}$ Department of Neuroscience, Tor Vergata University, Rome, Italy; ${ }^{3}$ Neuroimaging Laboratory, Santa Lucia Foundation, IRCCS, Rome, Italy
}

\begin{abstract}
In animal models of Alzheimer's disease (AD), mechanisms of cortical plasticity such as long-term potentiation (LTP) and long-term depression (LTD) are impaired. In AD patients, LTP-like cortical plasticity is abolished, whereas LTD seems to be preserved. Dopaminergic transmission has been hypothesized as a new player in ruling mechanisms of cortical plasticity in AD. We aimed at investigating whether administration of the dopamine agonist rotigotine (RTG) could modulate cortical plasticity in AD patients, as measured by theta burst stimulation (TBS) protocols of repetitive transcranial stimulation applied over the primary motor cortex. Thirty mild AD patients were tested in three different groups before and after 4 weeks of treatment with RTG, rivastigmine (RVT), or placebo (PLC). Each patient was evaluated for plasticity induction of LTP/LTD-like effects using respectively intermittent TBS (iTBS) or continuous TBS protocols. Short-latency afferent inhibition (SAI) protocol was performed to indirectly assess central cholinergic activity. A group of age-matched healthy controls was recruited for baseline comparisons. Results showed that at baseline, AD patients were characterized by impaired LTP-like cortical plasticity, as assessed by iTBS. These reduced levels of LTP-like cortical plasticity were increased and normalized after RTG administration. No effect was induced by RVT or PLC on LTP. LTD-like cortical plasticity was not modulated in any condition. Cholinergic activity was increased by both RTG and RVT. Our findings reveal that dopamine agonists may restore the altered mechanisms of LTP-like cortical plasticity in AD patients, thus providing novel implications for therapies based on dopaminergic stimulation.
\end{abstract}

Neuropsychopharmacology (2014) 39, 2654-266I; doi:I0.1038/npp.20 I4.II9; published online I8 June 2014

\section{INTRODUCTION}

In the case of Alzheimer's disease (AD), synaptic loss is the strongest pathophysiological correlate of cognitive decline, indicating that synaptic degeneration has a central role in the development of dementia (Klyubin et al, 2008). Experimental animal models showed that accumulation of soluble $\mathrm{A} \beta$ oligomers specifically block mechanisms of cortical plasticity such as hippocampal long-term potentiation (LTP) (Shankar et al, 2008) which is regarded as an electrophysiological correlate of learning and memory (Palop and Mucke, 2010). In contrast, these oligomers have been shown to electrically facilitate evoked long-term depression (LTD) (Li et al, 2009). These events can in turn induce changes in conformation of tau proteins, leading to further detrimental effects on synaptic plasticity and cognition (Boekhoorn et al, 2006). Similar mechanisms of cortical plasticity can be investigated in vivo and non-

* Correspondence: Dr G Koch, Non Invasive Brain Stimulation Unit, Laboratorio di Neurologia Clinica e Comportamentale, IRCCS Fondazione, S. Lucia, Via Ardeatina, Rome 306-00179, Italy,

Tel: +39065।501।81, Fax: +390651501।80,

E-mail: giakoch@gmail.com

Received I April 20I4; revised 6 May 20I4; accepted 19 May 20I4; accepted article preview online 26 May 2014 invasively in humans, although the plasticity-induction procedures adopted are not completely identical in humans and animals. In humans, LTP- and LTD-like measures may be obtained by applying repetitive transcranial magnetic stimulation (rTMS) over the primary motor cortex (M1), through protocols of theta burst stimulation (TBS) mimicking those described in animal models (Huang et al, 2005). In particular, 600 stimuli of continuous TBS (cTBS) are known to induce reliable LTD-like after-effects, whereas intermittent TBS (iTBS) can be used to obtain the opposite LTP-like effect (Huang et al, 2005). In AD patients, LTP-like cortical plasticity has been reported to be severely dampened when investigating M1 (Koch et al, 2012). This brain area is not severely affected by AD pathology, at least in its early clinical stages. For this reason, M1 can be used as a reliable model to assess cortical plasticity before the occurrence of diffuse synaptic dysfunction and gray matter loss, which instead are typically observed at later stages of the disease (ie, fully developed dementia) (Suvà et al, 1999). Additionally, a strict association has been reported between the levels of Tau protein found in the cerebro-spinal fluid of $\mathrm{AD}$ patients and the strength of cortical plasticity (Koch et al, 2011).

Dopamine is a key neuromodulator affecting several distinct steps of synaptic transmission including the probability of neurotransmitter release, the postsynaptic sensitivity to neurotransmitter and the membrane excitability of the 
pre- and postsynaptic cells (Tritsch and Sabatini, 2012). Its strong impact on motor cortical plasticity has been documented in both healthy individuals (Kuo et al, 2008; Monte-Silva et al, 2009) and patients with neurodegenerative disorders such as Parkinson's disease (Huang et al, 2011; Kishore et al, 2012). There is experimental evidence demonstrating that in the cerebral cortex, as well as in the basal forebrain, dopamine modulates the activity not only of pyramidal cells and GABA interneurons, but also of diffuse cholinergic projections from neurons located in the basal forebrain (Paspalas and Goldman-Rakic, 2005; Zhang et al, 2009). Notably, the dysfunction of dopaminergic transmission has been hypothesized as a new player in the pathophysiology of AD (Joyce et al, 1998; Kemppainen et al, 2003; Kumar and Patel, 2007; Martorana et al, 2010). In animal models of $\mathrm{AD}$, dopamine agonists may improve memory and even reduce intraneuronal amyloid deposition (Himeno et al, 2011). Localization studies of dopamine receptors in $\mathrm{AD}$ brains have shown a preferential reduction of dopamine D2-like receptors in the hippocampus and in the prefrontal cortex (Kemppainen et al, 2003; Kumar and Patel, 2007). Moreover, recent TMS studies performed over $\mathrm{M} 1$ revealed that in $\mathrm{AD}$ patients the impaired cholinergic transmission, measured by short-latency afferent inhibition (SAI) protocol (Di Lazzaro et al, 2002), can be transiently restored by the administration of Levodopa (Martorana et al, 2009). Similar effects can also be observed with rotigotine (RTG), a dopamine D2/D3 agonist (Martorana et al, 2013). Taken altogether, these data impose a careful investigation of the potential role of dopaminergic networks in mediating the synaptic efficiency/dysfunction in $\mathrm{AD}$. In this study, we aimed at investigating the impact of D2/D3 agonist RTG on cortical plasticity in patients suffering from mild AD. We hypothesized that dopaminergic stimulation would increase the altered mechanisms of LTP-like cortical plasticity that characterize AD patients (Koch et al, 2012). After recruitment and baseline investigation of cortical plasticity, patients were randomly assigned to three experimental arms: RTG, rivastigmine (RVT) or placebo (PLC). After 4 weeks, patients were reassessed for cortical plasticity.

\section{MATERIALS AND METHODS}

\section{Subjects}

Thirty consecutive patients with a diagnosis of probable $\mathrm{AD}$ according to the NINCDS-ADRDA Criteria (Varma et al, 1999) and 10 age-, sex- and education-matched healthy subjects (HS) were recruited for this study (Supplementary Table S1). All subjects underwent an extensive clinical investigation, including interviews on their medical history, a full neurological examination, the MMSE, a complete blood screening, neuropsychological assessment, neuropsychiatric evaluation, and magnetic resonance imaging. In the 30 days before entering the study, subjects had to be clear of treament with any drug inducing modulatory effects on the cerebral cortex excitability, such as antidepressants, benzodiazepines, anti-epileptic drugs, or neuroleptics. All AD patients had to show a moderate level of dementia, as assessed by a neuropsychological evaluation including the MMSE (ranging from 18 to 24) and a standardized neuropsychological battery (see below). Subjects were excluded if they had either two or more hyperintense lesions with a diameter $\geqslant 10 \mathrm{~mm}$ or more than eight hyperintense lesions with a diameter between 5 and $9 \mathrm{~mm}$ on dual-echo MR images (Bozzali et al, 2011). All participants (or their legal guardian in the case they were uncapable) signed a written informed consent after receiving an extensive disclosure of the experimental details. The current study was performed according to the Declaration of Helsinki and was approved by the Ethics Committee of Santa Lucia Foundation IRCSS in Rome (Prot. CE/AG4/PROG.392-08).

\section{Transcranial Magnetic Stimulation}

Motor-evoked potentials (MEPs) were recorded from the right first dorsal interosseous (FDI) muscles using 9-mm diameter, Ag-AgCl surface cup electrodes. Responses were amplified with a Digitimer D360 amplifier (Digitimer, UK) through filters set at $20 \mathrm{~Hz}$ and $2 \mathrm{kHz}$, then recorded by computer using SIGNAL software, at a sampling rate of $5 \mathrm{kHz}$ per channel (Cambridge Electronic Devices, UK). A monophasic Magstim 200 device (Magstim, UK) was used to define the motor hot spot in the left hemisphere and to assess MEP size. The motor hot spot was defined as the location where TMS consistently produced the largest MEP size at $120 \%$ of resting motor threshold in the target muscle (Rossini et al, 1994). A second coil was connected to a biphasic Super Rapid Magstim stimulator (Magstim) to deliver rTMS. In the cTBS protocol bursts at $80 \%$ active motor threshold were repeated at $5 \mathrm{~Hz}$ (ie, every $200 \mathrm{~ms}$ ), whereas each burst consisted of three stimuli repeating at $50 \mathrm{~Hz}$, for $40 \mathrm{~s}$ (600 pulses). In the iTBS protocol, a $2 \mathrm{~s}$ train of TBS was repeated 20 times, every $10 \mathrm{~s}$ for a total of $190 \mathrm{~s}$ (600 pulses) (Huang et al, 2005). Twenty MEPs were collected and averaged at baseline. Then, over the same hot spot, 20 MEPs were recorded at 1-5, 6-10, 11-15, 16-20, and 21-25 min after rTMS, and averaged. Although the LTP-like effects can be still pronounced after this interval, recording of MEP amplitude was stopped after $25 \mathrm{~min}$ in order to account for patients' compliance. SAI was investigated using the technique that has been recently described (Sailer et al, 2003), where the conditioning stimulus (CS) is a single pulse $(200 \mu \mathrm{s})$ of electrical stimulation applied through bipolar electrodes to the right median nerve at the wrist (cathode proximal). The intensity of the CS was set at just over motor threshold for evoking a visible twitch of the thenar muscles. The intensity of the cortical magnetic test stimulus (TS) was adjusted to evoke a muscle response in the relaxed right FDI with an amplitude of about $1 \mathrm{mV}$ peak-to-peak. The CS to the peripheral nerve preceded the cortical magnetic TS by different interstimulus intervals (ISI), that ranged from -4 to $+8 \mathrm{~ms}$ from the N20 component, in steps of $4 \mathrm{~ms}$ (Martorana et al, 2013). Ten stimuli were delivered at each ISI. Subjects were provided with audiovisual feedback at high gain, in order to assist them in maintaining complete relaxation. The inter-trial interval was set at $5 \mathrm{~s}( \pm 10 \%)$, for a total duration of $\sim 5$ min. Measurements were made on each individual trial. The mean peak-to-peak amplitude of the conditioned MEP at each ISI was expressed as a percentage of the mean peak-to-peak amplitude of the unconditioned test pulse in that block. 


\section{Cognitive Assessment}

All AD patients underwent a neuropsychological examination including a general cognitive index (Mini Mental Examination State), and the following neuropsychological tests for the specific cognitive domains (Carlesimo et al, 1996): (1) 15 Rey's Word List (immediate and 15-min delayed recall) to investigate verbal episodic long-term memory; (2) forward and backward Digit span and the forward and backward Corsi Block Tapping task to investigate short-term memory and working memory; (3) frontal assessment battery (FAB) to investigate executive functions (Apollonio et al, 2005). Parallel versions were used in the different sessions.

\section{Drug Administration and Experimental Design}

After recruitment and baseline assessments, AD patients were assigned to RTG $(n=10)$, RVT $(n=10)$ or PLC $(n=10)$. Physicians involved in TMS recordings and cognitive assessments were completely blind on which experimental branch each patient was belonging to. All treatments and PLC were administered for 4 weeks with no interruptions. RTG was administered through a $4 \mathrm{mg}$ transdermal patch (Neupro, UCB pharma), after having started with a $2 \mathrm{mg}$ patch for 1 week. We chose this scheme because such a drug has been previously found to be effective in modulating cholinergic activity in $\mathrm{AD}$ patients (Martorana et al, 2013). We chose to test the effects of RTG, which has an impact only on a subset of dopamine receptors, and not those of levo-dopa, which has a more global DA activation, because RTG can be administered with transdermal patches and it is well tolerated in $\mathrm{AD}$ patients (Martorana et al, 2013). Domperidone was administered in the case of dizziness occurrence. RVT was administered using Exelon Patch $4.6 \mathrm{mg}$. PLC was administered using a transdermal patch similar to those medicated. In all cases, each daily patch was maintained for $24 \mathrm{~h}$. The Monday before starting drug/PLC administration, and the Friday at the end of 4-week treatment, AD patients underwent TMS recordings and cognitive assessment. There was an interval of at least $1 \mathrm{~h}$ between the different TMS protocols. The order of TMS protocols was pseudorandomized in the different sessions.

For further follow-up evaluation, seven patients in the RTG group were kept on the same drug administration (4 mg transdermal patch) for 8 additional weeks. In these patients, another evaluation was performed on the Monday following the end of treatment, and reassess by TMS and nueropsychological testing the Friday of the 12th week.

\section{Data Analysis}

Data were analyzed using SPSS for Windows version 11.0; we measured the percentage of change of peak-to-peak amplitudes of the mean MEPs at baseline for each subject in each condition. At baseline two-way repeated measure ANOVAs were performed for each protocol (cTBS, iTBS) with TIME (1-5, 6-10, 11-15, 16-20, and 21-25 min after TBS) as within subjects factor and GROUP (RTG, RVT, PLC, HS) as between subjects factor. To test the effects of the drugs, different repeated measure ANOVAs were performed for each protocol (cTBS, iTBS) with SESSION (baseliine $v s$ end of treatment), TIME $(1-5,6-10,11-15,16-20$, and $21-$ $25 \mathrm{~min}$ after TBS) as within subjects factors and GROUP (RTG, RVT, PLC) as between subjects factor. For the SAI recordings, the electrophysiological parameters of $\mathrm{AD}$ patients were compared at baseline with those of agematched controls by means of repeated measures ANOVA, with ISI $(-4,0,+4$ and $+8 \mathrm{~ms}$ plus the latency of the N20) as within subject factor and GROUP (RTG, RVT, PLC $v s$ HS) as between subjects factor. To test the effects of the drugs, different repeated measure ANOVAs were performed with SESSION (before $v s$ end of treatment) and ISI $(-4,0$, +4 and $+8 \mathrm{~ms}$ plus the latency of the N20) as within subjects factors and GROUP (RTG, RVT, PLC) as between subjects factor. The Greenhouse-Geisser correction was used for nonspherical data. When a significant main effect was reached, paired $t$-tests with Bonferroni correction $(p<0.05)$ were used to characterize the different effects in all experiments. Mauchley's test examined for sphericity. To compare the effects of RTG, RVT, and PLC on cognitive performance before and after the treatment, paired $t$-tests were performed separately for each test. For all statistical analyses, a $p$ value $<0.05$ was considered to be significant.

\section{RESULTS}

The entire procedure was well tolerated in all subjects. The mean (SD) AMT (calculated as a percentage of maximal stimulator output) for TMS was significantly lower in $\mathrm{AD}$ patients (RTG: 34.2\%; RVT: 33.8\%; PLC: $35.2 \%$ ) compared with HS (38.2\%). Baseline MEP amplitudes did not differ between $A D$ subgroups (RTG $(1.12 \pm 0.41 \mathrm{mV}$ ), RVT $(1.09 \pm 0.42 \mathrm{mV})$, PLC $(1.21 \pm 0.42 \mathrm{mV}))$, and HS $(1.07 \pm 0.45 \mathrm{mV})$.

At baseline, for the cTBS protocol a repeated measures ANOVA was performed on the percentage changes of the mean MEP amplitude: no effect was seen for the GROUP $(\mathrm{F}(3,34)=0.96 ; p=0.42)$ and $\operatorname{TIME}(\mathrm{F}(4,136)=0.52 ; p=0.71)$ main factors as was not significant $(\mathrm{F}(12,136)=0.46 ; p=0.92)$ the interaction GROUP $\times$ TIME (Figure 1a). For the iTBS protocol, there was a significant GROUP $(\mathrm{F}(3,34)=17.02$; $p=0.0001$; RTG $v s$ HS: Cohen's $d=0.81$; RVT $v s$ HS: Cohen's $d=0.74$; PLC $v s$ HS Cohen's $d=0.41$ ) but not TIME $(\mathrm{F}(4,136)=1.26 ; p=0.28)$ main factor effect; the interaction GROUP $\times$ TIME was significant $(\mathrm{F}(12,136)=2.26 ; p=0.01)$, revealing that $\mathrm{AD}$ patients showed the expected altered LTPlike cortical plasticity in comparison with HS (Figure 1b). Accordingly, only in the HS group mean MEPs amplitudes were increased in comparison with baseline at 6-10, 11-15; $16-20$ and $21-25 \mathrm{~min}$. (all $p<0.05$ at paired $t$-test analyses). The ANOVA analysis performed on SAI protocol at baseline showed a significant ISI $(F(2,102)=31.3 ; p=0.029)$ but not GROUP $(\mathrm{F}(3,34)=0.60 ; p=0.61)$ main factor; the interaction GROUP $\times$ TIME was not significant $(\mathrm{F}(9,102)=0.95$; $p=0.48)$ (Figure 2).

The different drugs (RTG, RVT, PLC) did not induce any effect on the cTBS protocol as shown by the ANOVA (GROUP main factor: $\mathrm{F}(2,25)=0.20 ; p=0.81$; SESSION main factor: $\mathrm{F}(1,25)=65.38 ; p=0.007$; TIME main factor: $\mathrm{F}(4,100)=0.42 ; p=0.78$; interaction GROUP $\times$ SESSION $\times$ TIME: $\mathrm{F}(8,100)=0.70 ; p=0.75$; Figure $3 \mathrm{a}$ ). On the other hand for the iTBS protocol, RTG induced an increase of 

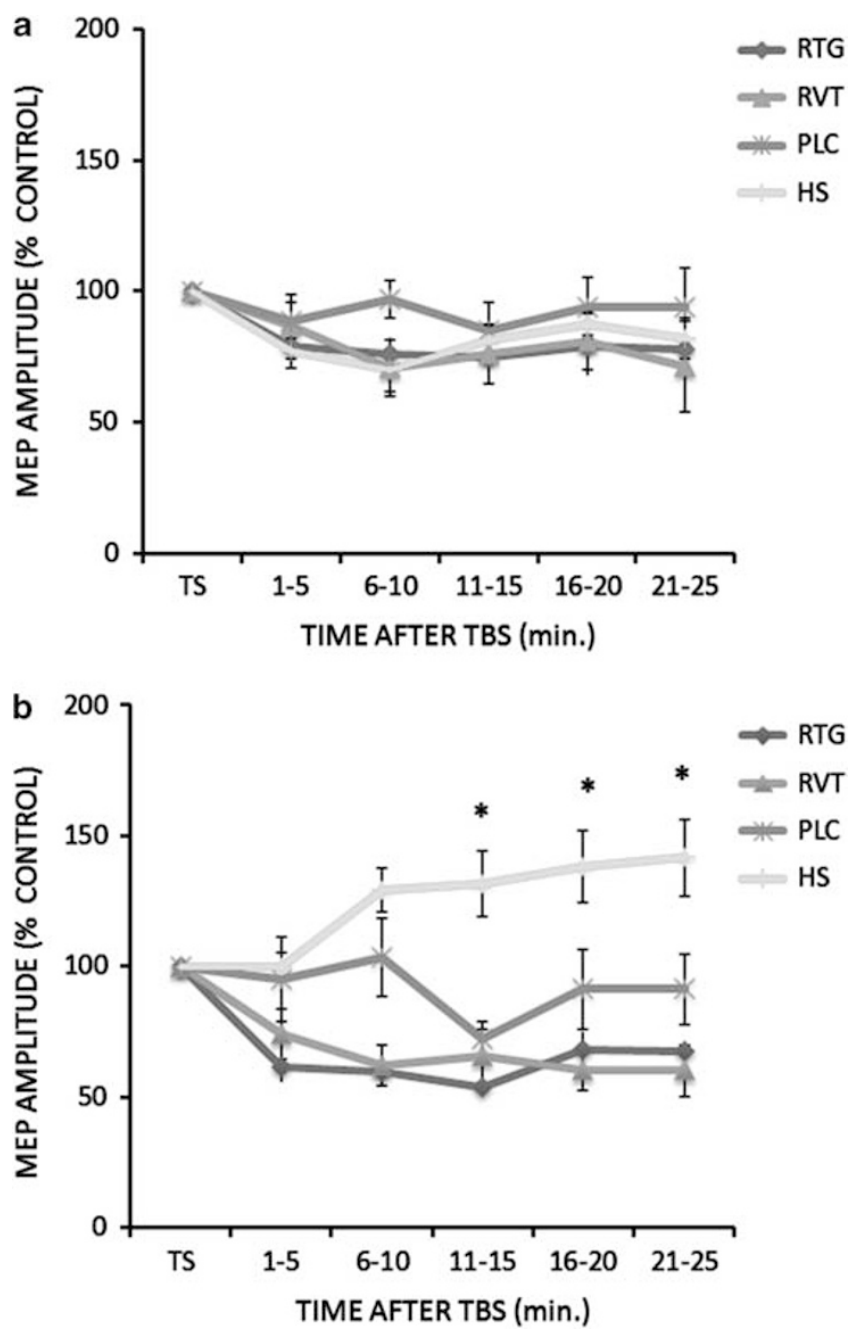

Figure I After effects of the CTBS (a) and iTBS (b) protocols on MEP amplitude in the different groups of Alzheimer's disease patients at baseline before starting the treatment with RTG, RVT, and PLC. A group of agematched HS was included for comparison at baseline only. Error bars indicate SEM. Asterisks indicate $p<0.05$ when comparing AD patients of the different groups.

LTP-like cortical plasticity in $\mathrm{AD}$ patients, as revealed by the ANOVA showing significant GROUP $(\mathrm{F}(2,25)=51.96$; $p=0.00004$; RTG vs PLC: Cohen's $d=0.81$; RTG vs RVT: Cohen's $d=0.69)$ and SESSION $(\mathrm{F}(1,25)=103.73$; $p=0.000001)$ main factors and GROUP $\times$ SESSION $(\mathrm{F}(2,25)$ $=74.7 ; p=0.000001)$ and GROUP $\times \operatorname{TIME}((8,100)=23.4$; $p=0.02)$ interactions. The TIME main factor $(\mathrm{F}(4,100)=0.24$; $p=0.88)$ and the other interactions were not significant $($ TIME $\times$ SESSION $\quad(\mathrm{F}(4,100)=19.2 ; \quad p=0.11) ; \quad$ TIME $\times$ GROUP $\times$ SESSION $\quad(\mathrm{F}(8,100)=12.62 ; p=0.27))$. Post hoc analysis with Bonferroni correction showed that LTP increased in the RTG group only $(p<0.0001$ at all time points in comparison with the other AD groups) (Figure $3 b$ ). Accordingly, MEPs amplitudes were increased in comparison with baseline only in the RTG group at 1-5, 6-10, 11-15,16-20 and 21-25 min. (all $p<0.05$ at paired $t$-test analyses).

For the SAI protocol, the ANOVA showed significant ISI main factor $(\mathrm{F}(3,75)=6.63 ; p=0.00038)$, ISI $\times$ SESSION interaction $(\mathrm{F}(3,75)=23.70 ; p=0.0001)$ and GROUP $\times$

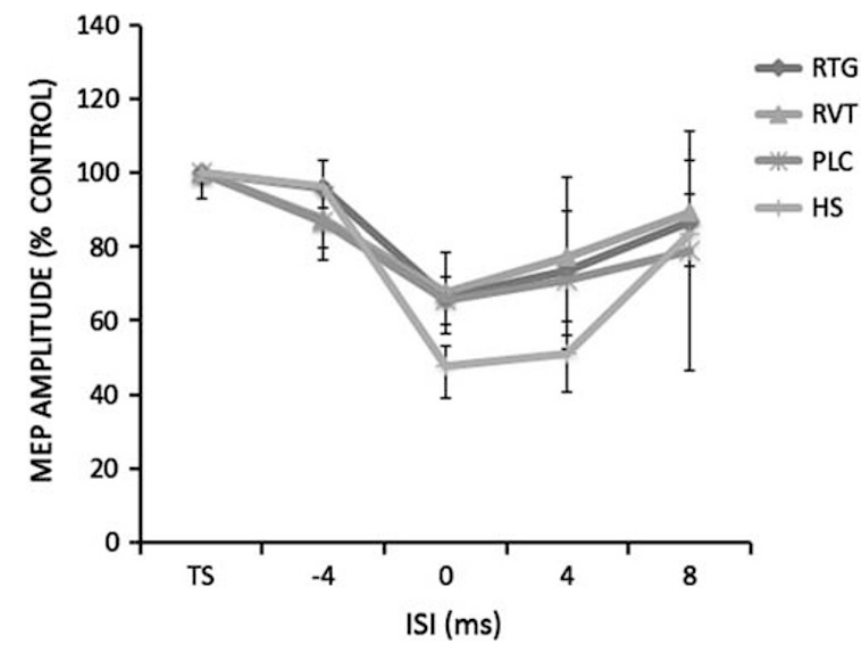

Figure 2 After effects of the SAI protocol on MEP amplitude in the different groups of Alzheimer's disease patients at baseline before starting the treatment with RTG, RVT, and PLC. A group of age-matched HS was included for comparison at baseline only. Error bars indicate SEM. Asterisks indicate $p<0.05$ when comparing $A D$ patients of the different groups.
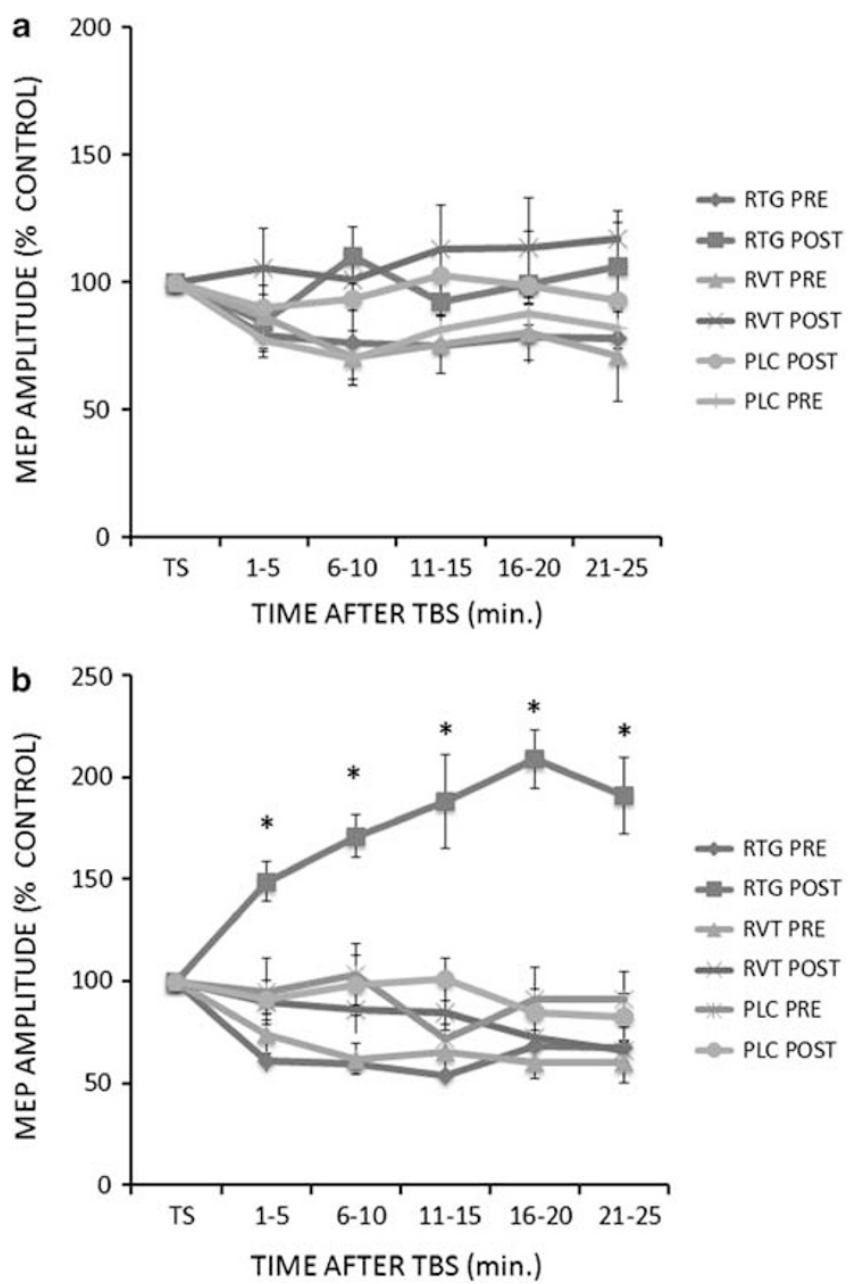

Figure 3 After effects of the cTBS (a) and iTBS (b) protocols on MEP amplitude in Alzheimer's disease patients at baseline (pre) and after 4 weeks (post) of treatment with RTG, RVT, and PLC. Error bars indicate SEM. Asterisks indicate $p<0.05$ when comparing AD patients of the different groups. 


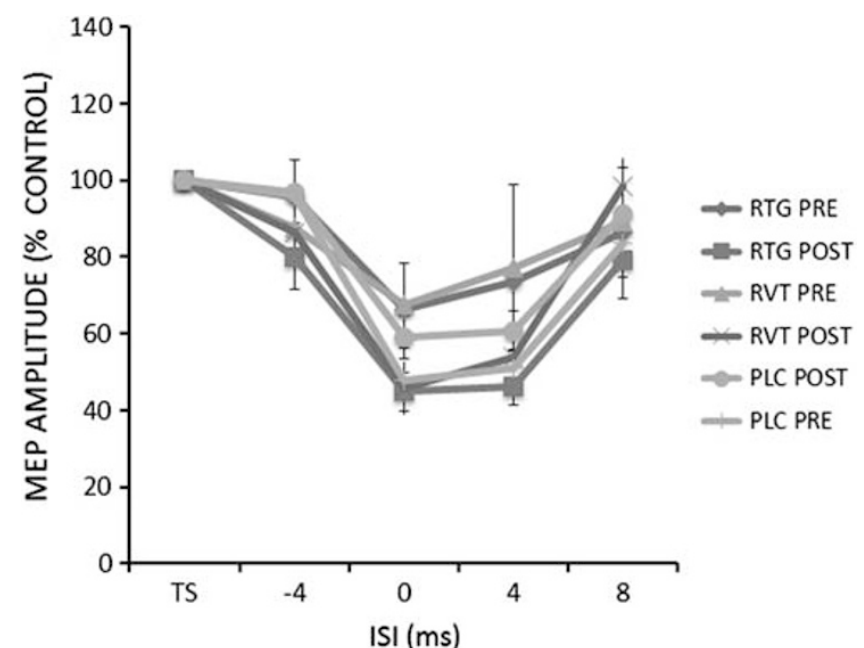

Figure 4 After effects of the SAI protocols on MEP amplitude in Alzheimer's disease patients at baseline (pre) and after 4 weeks (post) of treatment with RTG, RVT, and PLC. Error bars indicate SEM. Asterisks indicate $p<0.05$ when comparing AD patients of the different groups.

SESSION interaction $(\mathrm{F}(2,25)=3.27 ; p=0.04))$. Post hoc analysis showed that SAI was increased in comparison with baseline at ISI $=0 \mathrm{~ms}$ in both RTG and RVT groups (Figure 4).

For the seven $\mathrm{AD}$ patients retested after 12 weeks, the analysis performed on the cTBS data did not show any effect for both the SESSION $(\mathrm{F}(2,12)=12.2 ; p=0.07)$, and the TIME main factors $(\mathrm{F}(4,24)=26.31 ; p=0.06)$, and the SESSION $\times$ TIME interaction $\quad(\mathrm{F}(8,48)=20.3 ; \quad p=0.11)$ (Figure 5a). For the iTBS protocol, there was a significant SESSION $(\mathrm{F}(2,12)=94.3 ; p=0.0000001)$ but not TIME $(\mathrm{F}(4,24)=13.7 ; p=0.16)$ main factor effect. The SESSION $\times$ TIME interaction $(\mathrm{F}(8,48)=16.86 ; p=0.012)$ was significant. Post hoc analysis with Bonferroni correction showed that LTP increased in the RTG group in comparison with baseline evaluation at 4 weeks at all time points $(p<0.001)$ and at 12 weeks at $1-5,6-10,11-15$ and 16-20 min (all $p<0.01$ ) (Figure $5 b$ ). For the SAI data, ANOVA revealed significant SESSION $(\mathrm{F}(2,12)=44.08 ; p=0.0001)$, ISI $(\mathrm{F}(3,18)=3.25 ; p=0.046)$ and SESSION $\times$ ISI interaction $(\mathrm{F}(6,36)=4.46 ; p=0.017)$. Post hoc analyses showed that SAI was increased in comparison with baseline at ISI $=0 \mathrm{~ms}$ after 4 and 12 weeks of treatment with RTG $(p$ all $<0.05)$ (Figure 5c).

To control for any change in cognitive functions, neuropsychological scores obtained at baseline were compared with those obtained after 4 weeks of treatment. MMSE, RAVLT, Digit span, Corsi span, and FAB scores at baseline did not differ between the three AD subgroups. The repeated measure ANOVAs performed on the mean scores for each different cognitive test before and after tretament between the three groups of $\mathrm{AD}$ patients did not reveal any difference. However paired $t$-test analysis comparing within each group, the different cognitive scores before and after tretament showed that in the RTG group there was an improvement of MMSE scores $(t(9)=2.65, p<0.05)$ and an improvement of the FAB scores $(t(9)=2.47, p<0.05)$. There was no correlation between the individual percentage of change in the MMSE and the FAB scores and the individual
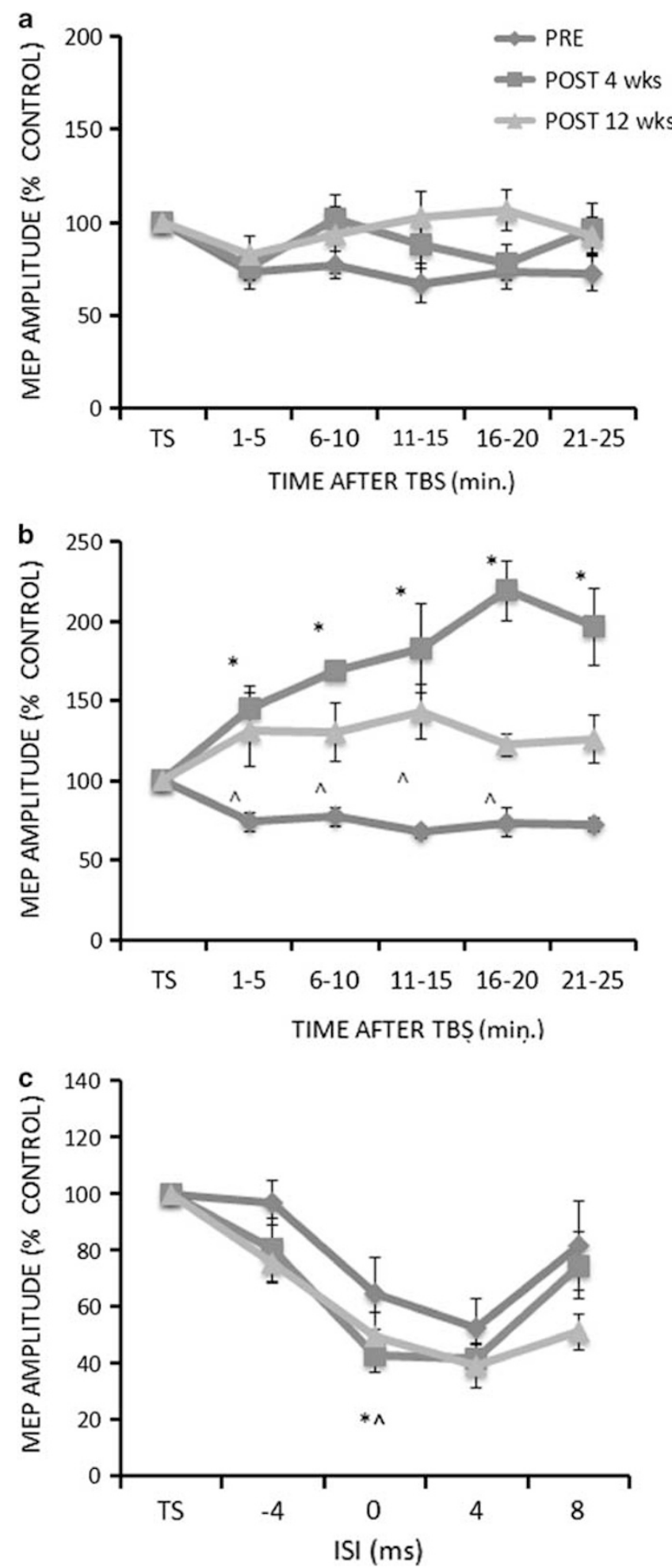

Figure 5 After effects of the cTBS (a) and iTBS (b) protocols on MEP amplitude in the RTG Alzheimer's disease patients group at baseline (pre), after 4 and 12 weeks of treatment with RTG. (c) After effects of the SAl protocols on MEP amplitude in the RTG Alzheimer's disease patients group at baseline (pre), after 4 and 12 weeks of treatment with RTG. The figure shows the results of the subgroup of patients $(n=7)$ involved in the whole 12 weeks course. Error bars indicate SEM. * $p<0.05$ between the 4 weeks and the baseline evaluation. ${ }^{\wedge} p<0.05$ between the 12 weeks and the baseline evaluation. 
mean percentage change of MEP amplitude for the iTBS protocol before and after RTG treatment (MMSE: $r=0.21$; $p=$ n.s.; FAB: $\mathrm{r}=-0.28 ; p=$ n.s.). In the RVT group there was an improvement in the MMSE scores $(t(9)=2.36 p<0.05)$ (Supplementary Table S2), whereas no changes of MMSE scores $(t(9)=-0.07, p=0.94)$ and FAB scores $(t(9)=-1.93$, $p=0.09$ ) were observed in the PLC group. For the cognitive performances of seven $\mathrm{AD}$ patients of the RGT group that were followed up at 12-week paired $t$-tests showed that there were improvements in the MMSE $(t=(6) 2.71, p<0.05)$ and FAB $(t(6)=2.57 ; p<0.05)$ (Supplementary Table S3).

\section{DISCUSSION}

Our study demonstrates that the dopamine agonist RTG has the ability to restore altered mechanisms of LTP-like cortical plasticity in AD patients.

The increase of LTP-like cortical plasticity is in line with the notion that dopamine is a key player in modulating cortical plasticity in healthy individuals (Kuo et al, 2008; Monte-Silva et al, 2009) and in patients with Parkinson's disease (Huang et al, 2011; Kishore et al, 2012). It is indeed well established from animal models that dopamine D1receptor activation prolongs LTP and LTD (Otmakhova and Lisman, 1998). Conversely, positive and negative effects can both be identified on LTP and LTD when stimulating D2/D3 receptors (Manahan-Vaughan and Kulla, 2003) indicating that dopamine has a non uniform excitatory or inhibitory function. In this view, dopamine can rather act as a strong neuromodulator, with the ability of inducing an effect on cortical activity and plasticity by changing the signal-tonoise ratio (Seamans and Yang, 2004). This focusing effect was confirmed in humans by use of neuroplasticityinducing stimulation paradigms, namely transcranial direct current stimulation and paired associative stimulation (PAS), which elicit time-dependent LTP- and LTD-like effects on cortical excitability (Kuo et al, 2008). An appropriate balance of D1 and D2 activity seems therefore necessary to elicit a focusing effect (Nitsche et al, 2009). D2like receptor activation produces an inverted ' $U$ '-shaped dose-response curve on plasticity, supporting the assumption that modulation of D2-like receptor activity exerts dose-dependent inhibitory or facilitatory effects on neuroplasticity in the human motor cortex (Monte-Silva et al, 2009). Crucially, a recent study examined effects of D2 receptor blockade sulpiride on the LTP/LTD-like effects of TBS. Administration of sulpiride blocked the effects of both iTBS and CTBS, showing that D2 receptors are strongly involved in the generation of LTP/LTD-like effects mediated by TBS (Monte-Silva et al, 2011). Dopaminergic stimulation is also effective in modulating TBS effects in pathological conditions in which dopaminergic depletion occurs, such as Parkinson's disease. These patients may present with normal LTP-like effects when they assume a full dose of Levodopa (Huang et al, 2011). In contrast, there is no LTPlike effect when they are administered half a dose of Levodopa (Huang et al, 2011). Moreover, these beneficial boosting effects on plasticity convert to a negative effect as disease advances (Kishore et al, 2012).

Our data indicate that a similar boosting effect can be detected also in patients with mild AD. Notably, our AD patients were treated with a RTG dosage of $4 \mathrm{mg}$, which is considered as a relatively low dose. Further studies are needed to clarify whether an increased dose of RTG could further increase or even reduce the effects on LTP-like cortical plasticity resembling the inverted ' $U$ '-shaped doseresponse curve on plasticity described in healthy controls (Monte-Silva et al, 2009). One might argue that the dopaminergic effects on cortical plasticity may be ascribed to an interplay with the cholinergic transimission. On this issue, it was recently demonstrated that, in patients with mild $\mathrm{AD}$, the administration of both Levodopa and RTG is able to increase the levels of central cholinergic activity as assessed by SAI (Martorana et al, 2009, 2013). In the current study, the main analyses of SAI measurements did not show any difference between RTG and RVT. This is in line to what expected as both drugs are known to increase SAI activity in AD (Di Lazzaro et al, 2002; Marotrana et al, 2009; Monte-Silva et al, 2011; Di Lazzaro et al, 2005). This might suggest that the effects on cortical plasticity induced by RTG administration are specifically driven by dopaminergic stimulation.

A role for the dopaminergic system in $\mathrm{AD}$ brains has long been sought and it is still debated. Post mortem studies revealed marked loss of dopamine receptors in the temporal and frontal lobes of AD brains (Kemppainen et al, 2003; Kumar and Patel, 2007; Martorana et al, 2010), regions classically involved in cognitive decline. Interestingly, most of the changes regarding the dopamine receptors, particularly the D2 subtype, were found at rostral and mid-levels of the temporal cortex, indicating that regions classically affected by $\mathrm{AD}$ pathology are also sensitive to the loss of $\mathrm{D} 2$ receptors (Joyce et al, 1998; Goldsmith and Joyce, 1996; Joyce et al, 1993; Ryoo and Joyce, 1994). These dopamine D2/D3 receptors may have an important role in the reciprocal activity of large groups of neurons in the high-order association cortical regions, and may promote the cognitive and behavioural impairments observed in $\mathrm{AD}$. Using antipeptide rabbit antibodies for each of the five dopamine receptors (D1-D5), Kumar and Patel (2007) mapped their distribution in the frontal cortex of post mortem AD brains and reported a severe reduction of of D1 receptor, D3 receptor, and D4 receptor expression. Conversely, the D2 receptor expression was only moderately reduced. This pattern could explain why the administration of the D2 agonist exerted such a powerful effect on cortical plasticity in our sample of $\mathrm{AD}$ patients. Despite defective, the activity of D2 receptors is likely to be potentiated by increasing the levels of D2 agonists. In another series of studies, D2 receptors activity was evaluated in vivo by using PET. D2 receptor binding was significantly reduced in the striatum of $\mathrm{AD}$ patients, even in the absence of overt extra-pyramidal symptoms (Pizzolato et al, 1998). Kemppainen et al (2003) reported that $\mathrm{D} 2$ receptor binding potentials are reduced in the hippocampus by $30 \%$ in $\mathrm{AD}$ patients as compared with controls. This reduction was found to be associated with both cognitive (Kemppainen et al, 2003) and behavioral abnormalities in AD patients (Tanaka et al, 2003).

Dopamine is known to have an important role in higher cognitive functions such as memory, learning, attention, and decision making (Backman et al, 2006; Cole et al, 2011). Interestingly, animal and human studies have consistently demonstrated that an inverted- $U$ curve exists between 
dopaminergic signaling and cognition. Although low or high levels of dopamine impair cognition, middle dosages of dopamine appears necessary for optimal performances (Baunez and Robbins, 1999; Boussaoud and Kermadi, 1997; Cools and D'Esposito, 2011; Glickstein et al, 2005). This phenomenon somewhat resembles what has already been observed with cortical plasticity measurements (MonteSilva et al, 2009). Recent evidence revealed that, in animal models of $\mathrm{AD}$, dopamine agonists may improve memory function of transgenic-AD mices, inducing decreases in intraneuronal amiloid beta and tau protein deposition, strongly suggesting that dopaminergic agents could be a novel drug for $\mathrm{AD}$ (Himeno et al, 2011). However, the involvement of dopamine in the cognitive symptoms in patients with $\mathrm{AD}$ has been poorly investigated. Data are limited to indirect evidence that $\mathrm{AD}$ patients with higher MMSE scores have an increased capacity of striatal dopamine synthesis (Kemppainen et al, 2003; Tanaka et al, 2003). Notably in the RTG group, despite the clear effects on LTP-like cortical plasticity, we only found a trend towards some improvement for the frontal executive functions. Moreover, we did not find any correlation between the percentage of change of LTP-like cortical plasticity and the relative change of the scores obtained in the different cognitive tests after RTG administration. Clearly, randomized controlled trials are needed to determine whether dopamine agonists may have a clinical relevance in improving cognitive functions in $\mathrm{AD}$ patients. These drugs could involve the modulation of the dopaminergic mesocortico-limbic pathway that has important roles in reward, motivation, executive functions, learning, and memory (Kurniawan et al, 2011).

This study has some limitations. The fact that dopaminergic enhancement improved plasticity in these patients is not a definite proof of the causal pathophysiological involvement of the dopaminergic system in Alzheimer's disease. It could be argued that dopamine compensates for reduced cholinergic functions without being causally involved in pathophysiology. On the other hand, dopaminergic activity could be diminished secondarily owing to loss of other transmitters/ modulators. Moreover, measures of plasticity were obtained in the motor cortex, which is typically affected by $\mathrm{AD}$ pathology at late disease stages. Future studies should focus on the association cortex (eg, temporo-parietal junction or dorsolateral preforntal cortex) using different neurophysiological methods such as TMS in combination with EEG (Veniero et al, 2013; Rajji et al, 2013).

In conclusion, we demonstrated that the administration of dopamine agonists such as RTG is effective in modulating the altered mechanisms of cortical plasticity that characterize $\mathrm{AD}$ patients. This could open new perspectives for therapeutic strategies to contrast cortical dysfunction in $\mathrm{AD}$.

\section{FUNDING AND DISCLOSURE}

The authors declare no conflict of interest.

\section{ACKNOWLEDGEMENTS}

This work was supported by grants of the Italian Ministry of Health to GK (239/GR-2009-1591859) and to MB (47/RF2010-2311484).

\section{REFERENCES}

Appollonio I, Leone M, Isella V, Piamarta F, Consoli T, Villa ML et al (2005). The Frontal Assessment Battery (FAB): normative values in an Italian population sample. Neurol Sci 26: 108-116.

Backman L, Nyberg L, Lindenberger U, Li SC, Farde L (2006). The correlative triad among aging, dopamine, and cognition: current status and future prospects. Neurosci Biobehav Rev 30: 791-807.

Baunez C, Robbins TW (1999). Effects of dopamine depletion of the dorsal striatum and further interaction with subthalamic nucleus lesions in an attentional task in the rat. Neuroscience 92: 1343-1356.

Boekhoorn K, Terwel D, Biemans B, Borghgraef P, Wiegert O, Ramakers GJ et al (2006). Improved long-term potentiation and memory in young tau-P301L transgenic mice before onset of hyperphosphorylation and tauopathy. J Neurosci 26: 3514-3523.

Boussaoud D, Kermadi I (1997). The primate striatum: neuronal activity in relation to spatial attention versus motor preparation. Eur J Neurosci 9: 2152-2168.

Bozzali M, Padovani A, Caltagirone C, Borroni B (2011). Regional grey matter loss and brain disconnection across Alzheimer disease evolution.. Curr Med Chem 18: 2452-2458.

Carlesimo GA, Caltagirone C, Gainotti G (1996). The Mental Deterioration Battery: normative data, diagnostic reliability and qualitative analyses of cognitive impairment. The Group for the Standardization of the Mental Deterioration Battery. Eur Neurol 36: 378-384.

Cole DM, Beckmann CF, Searle GE, Plisson C, Tziortzi AC, Nichols TE et al (2011). Orbitofrontal connectivity with resting-state networks is associated with midbrain dopamine D3 receptor availability. Cereb Cortex 22: 2784-2793.

Cools R, D’Esposito M (2011). Inverted-U-shaped dopamine actions on human working memory and cognitive control. Biol Psychiatry 69: 113-125.

Di Lazzaro V, Oliviero A, Pilato F, Saturno E, Dileone M, Marra C et al (2005). Neurophysiological predictors of long term response to AChE inhibitors in AD patients. J Neurol Neurosurg Psychiatry 76: 1064-1069.

Di Lazzaro V, Oliviero A, Tonali PA, Marra C, Daniele A, Profice P et al (2002). Non invasive in vivo assessment of cholinergic cortical circuits in AD using transcranial magnetic stimulation. Neurology 59: 392-397.

Glickstein SB, Desteno DA, Hof PR, Schmauss C (2005). Mice lacking dopamine D2 and D3 receptors exhibit differential activation of prefrontal cortical neurons during tasks requiring attention. Cereb Cortex 15: 1016-1024.

Goldsmith SK, Joyce JN (1996). Dopamine D2 receptors are organized in bands in normal human temporal cortex. Neuroscience 74: 435-451.

Himeno E, Ohyagi Y, Ma L, Nakamura N, Miyoshi K, Sakae N et al (2011). Apomorphine treatment in Alzheimer mice promoting amyloid- $\beta$ degradation. Ann Neurol 69: 248-256.

Huang YZ, Edwards MJ, Rounis E, Bhatia KP, Rothwell JC (2005). Theta burst stimulation of the human motor cortex. Neuron 45: 201-206.

Huang YZ, Rothwell JC, Lu CS, Chuang WL, Chen RS (2011). Abnormal bidirectional plasticity-like effects in Parkinson's disease. Brain 134: 2312-2320.

Joyce JN, Kaeger C, Ryoo H, Goldsmith S (1993). Dopamine D2 receptors in the hippocampus and amygdala in Alzheimer's disease. Neurosci Lett 154: 171-174.

Joyce JN, Myers AJ, Gurevich E (1998). Dopamine D2 receptor bands in normal human temporal cortex are absent in Alzheimer's disease. Brain Res 784: 7-17.

Kemppainen N, Laine M, Laakso MP, Kaasinen V, Någren K, Vahlberg $\mathrm{T}$ et al (2003). Hippocampal dopamine D2 receptors correlate with memory functions in Alzheimer's disease. Eur J Neurosci 18: 149-154. 
Kishore A, Popa T, Velayudhan B, Joseph T, Balachandran A, Meunier S (2012). Acute dopamine boost has a negative effect on plasticity of the primary motor cortex in advanced Parkinson's disease. Brain 135: 2074-2088.

Klyubin I, Betts V, Welzel AT, Blennow K, Zetterberg H, Wallin A et al (2008). Amyloid beta protein dimer-containing human CSF disrupts synaptic plasticity: Prevention by systemic passive immunization. J Neurosci 28: 4231-4237.

Koch G, Esposito Z, Kusayanagi H, Monteleone F, Codecá C, Di Lorenzo F et al (2011). CSF Tau levels influence cortical plasticity in Alzheimer's disease patients. J Alzheimers Dis 26: 181-186.

Koch G, Di Lorenzo F, Bonnì S, Ponzo V, Caltagirone C, Martorana A (2012). Impaired LTP- but not LTD-like cortical plasticity in Alzheimer's disease patients. J Alzheimers Dis 31: 593-599.

Kumar U, Patel SC (2007). Immunohistochemical localization of dopamine receptor subtypes (D1R-D5R) in Alzheimer's disease brain. Brain Res 1131: 187-196.

Kuo MF, Paulus W, Nitsche MA (2008). Boosting focally-induced brain plasticity by dopamine. Cereb Cortex 18: 648-651.

Kurniawan IT, Guitart-Masip M, Dolan RJ (2011). Dopamine and effort-based decision making. Front Neurosci 21: 81.

Li S, Hong S, Shepardson NE, Walsh DM, Shankar GM, Selkoe D (2009). Soluble oligomers of amyloid Beta protein facilitate hippocampal long-term depression by disrupting neuronal glutamate uptake. Neuron 62: 788-801.

Manahan-Vaughan D, Kulla A (2003). Regulation of depotentiation and long-term potentiation in the dentate gyrus of freely moving rats by dopamine D2-like receptors. Cereb Cortex 13: 123-135.

Martorana A, Mori F, Esposito Z, Kusayanagi H, Monteleone F, Codecà C et al (2009). Dopamine modulates cholinergic cortical excitability in Alzheimer's disease patients. Neuropsychopharmacology 34: 2323-2328.

Martorana A, Esposito Z, Koch G (2010). Beyond the cholinergic hypothesis:do current drugs work in Alzheimer's disease? CNS Neurosci Ther 16: 235-245.

Martorana A, Di Lorenzo F, Esposito Z, Lo Giudice T, Bernardi G, Caltagirone $C$ et al (2013). Dopamine $\mathrm{D}_{2}$-agonist rotigotine effects on cortical excitability and central cholinergic transmission in Alzheimer's disease patients. Neuropharmacology 64: 108-113.

Monte-Silva K, Kuo MF, Thirugnanasambandam N, Liebetanz D, Paulus W, Nitsche MA (2009). Dose-dependent inverted U-shaped effect of dopamine (D2-like) receptor activation on focal andnonfocal plasticity in humans. J Neurosci 29: 6124-6131.

Monte-Silva K, Ruge D, Teo JT, Paulus W, Rothwell JC, Nitsche MA (2011). D2 receptor block abolishes $\theta$ burst stimulationinduced neuroplasticity in the human motor cortex. Neuropsychopharmacology 36: 2097-2102.

Nitsche MA, Kuo MF, Grosch J, Bergner C, Monte-Silva K, Paulus W (2009). D1-receptor impact on neuroplasticity in humans. J Neurosci 29: 2648-2653.

Otmakhova NA, Lisman JE (1998). D1/D5 dopamine receptors inhibit depotentiation at CA1 synapses via cAMP-dependent mechanism. J Neurosci 18: 1270-1279.
Palop JJ, Mucke L (2010). Amyloid-beta-induced neuronal dysfunction in Alzheimer's disease: from synapses toward neural networks. Nat Neurosci 13: 812-818.

Paspalas CD, Goldman-Rakic PS (2005). Presynaptic D1 dopamine receptors in primate prefrontal cortex: target-specific expression in the glutamatergic synapse. J Neurosci 25: 1260-1267.

Pizzolato G, Chierichetti F, Fabbri M, Cagnin A, Dam M, Ferlin G et al (1998). Reduced striatal dopamine receptors in Alzheimer's disease: single photon emission tomography study with the D2 tracer [123I]-IBZM. Neurology 47: 1065-1068.

Rajji TK, Sun Y, Zomorrodi-Moghaddam R, Farzan F, Blumberger $\mathrm{DM}$, Mulsant BH et al (2013). PAS-induced potentiation of cortical-evoked activity in the dorsolateral prefrontal cortex. Neuropsychopharmacol 38: 2545-2552.

Rossini PM, Barker AT, Berardelli A, Caramia MD, Caruso G, Cracco RQ et al (1994). Non-invasive electrical and magnetic stimulation of the brain, spinal cord and roots: basic principles and procedures for routine clinical application. Report of an IFCN committee.. Electroencephalogr Clin Neurophysiol 91: 79-92.

Ryoo HL, Joyce JN (1994). Loss of dopamine D2 receptors varies along the rostrocaudal axis of the hippocampal complex in Alzheimer's disease. J Comp Neurol 348: 94-110.

Sailer A, Molnar GF, Paradiso G, Gunraj CA, Lang AE, Chen R (2003). Short and long latency afferent inhibition in Parkinson's disease. Brain 126: 1883-1894.

Seamans JK, Yang CR (2004). The principal features and mechanisms of dopamine modulation in the prefrontal cortex. Prog Neurobiol 74: 1-58.

Shankar GM, Li S, Mehta TH, Garcia-Munoz A, Shepardson NE, Smith I et al (2008). Amyloid-beta protein dimers isolated directly from Alzheimer's brains impair synaptic plasticity and memory. Nat Med 14: 837-842.

Suvà D, Favre I, Kraftsik R, Esteban M, Lobrinus A, Miklossy J (1999). Primary motor cortex involvement in Alzheimer disease. J Neuropathol Exp Neurol 58: 1125-1134.

Tanaka Y, Meguro K, Yamaguchi S, Ishii H, Watanuki S, Funaki Y et al (2003). Decreased striatal D2 receptor density associated with severe behavioral abnormality in Alzheimer's disease. Ann Nucl Med 17: 567-573.

Tritsch NX, Sabatini BL (2012). Dopaminergic modulation of synaptic transmission in cortex and striatum. Neuron 76: 33-50.

Varma AR, Snowden JS, Lloyd JJ, Talbot PR, Mann DM, Neary D. (1999). Evaluation of the NINCDS-ADRDA criteria in the differentiation of Alzheimer's disease and frontotemporal dementia. J Neurol Neurosurg Psychiatry 66: 184-188.

Veniero D, Ponzo V, Koch G (2013). Paired associative stimulation enforces the communication between interconnected areas. J Neurosci 33: 13773-13783.

Zhang L, Doyon WM, Clark JJ, Phillips PE, Dani JA (2009). Controls of tonic and phasic dopamine transmission in the dorsal and ventral striatum. Mol Pharmacol 76: 396-404.

Supplementary Information accompanies the paper on the Neuropsychopharmacology website (http://www.nature.com/npp) 